\title{
Numerical study of the detonation wave structure in ethylene-oxygen mixtures
}

\author{
A.M. Khokhlov \\ The University of Chicago, Chicago, IL 60637 \\ J.M. Austin, F. Pintgen, J.E. Shepherd \\ California Institute of Technology, Pasadena, CA 91125
}

\begin{abstract}
We examine a transition from a weakly to a highly unstable regime of a cellular detonation in stoichiometric ethylene-oxygen systems with varied dilution. The structure and propagation of cellular detonations is calculated using two-dimensional, time-dependent, reactive Euler fluid-dynamics algorithm. A dynamically adapting mesh is used to resolve reaction zones, shocks, contact surfaces, and vortices in flow. A simplified chemical model with Arrhenius kinetics is used for all mixtures. Effects of dilution are modeled by varying the adiabatic index $\gamma$ and molecular weight of matter. Results are compared to experimental data obtained in a detonation tube by simultaneous visualization of a chemical species $(\mathrm{OH})$, density gradients in the reaction zone, and to soot foil records. Due to a strong sensitivity of the post-shock temperature to variations of $\gamma$, the degree of chemical - fluid dynamics coupling inside the detonation structure varies significantly with dilution. Variations of the equation of state with dilution can account for and could be the main mechanism explaining a wide range of detonation behavior observed in the experiments.
\end{abstract}

\section{Introduction}

Modeling of gaseous detonation fronts is an extremely challenging problem due to non-linearity of the coupling between chemistry and fluid dynamics, the instability of the flow, and extraordinary range of length and time scales that are exhibited. A detonation front may show a regular or a highly irregular cellular behavior depending on the nature of a chemical system, and the nature and amount of a dilutent. In particular, ethylene-oxygen mixtures diluted with argon are weakly instable and display regular detonation cells, ${ }^{14}$ whereas diluted with nitrogen they may be highly unstable and show irregular behavior. ${ }^{1} \mathrm{Nu}-$ merical simulations using a simplified progress variable reaction models, detailed, or reduced chemical kinetics were able to reproduce regular detonation cells structure of weakly unstable detonations. ${ }^{2-8}$ Computations of irregular detonations are more challenging. Only recently strongly unstable detonations displaying secondary detonation cells were calculated in two dimensions using adaptive mesh refinement and a model Arrhenius reaction with a single progress variable. ${ }^{9,10}$

In this paper, we want to examine a transition from a weakly to a highly unstable regime of a cellular detonation in a stoichiometric ethylene-oxygen system with varied dilution. According to experimental observations and stability analyses, ${ }^{1,11}$ irregular behavior sets in a system when the reduced activation energy exceeds a certain value, $Q / R T_{s}>6$, where $Q$ is the dimensional activation energy, and $T_{s}$ is a temperature

Copyright (C) 2004 by The University of Chicago, Californis Institute of The American Institute of Anstitute of Technology. Published by the American behind the leading shock. ${ }^{1}$ As a first step we are using a model reaction with a single progress variable, Arrhenius kinetics, and an ideal gas constant heat capacity (constant $\gamma$ ) equation of state. Our goal is to examine the role of varying chemical instability on the transition from regular to irregular cellular structure due to variations in $T_{s}$ caused by changes in the equation of state of the system. The study combines numerical simulations of gaseous detonations and experimental visualizations of a detonation front structures for a variety of fuel-oxidizer systems with different degree of instability.

\section{Physical and Numerical Model}

The system is described by the time-dependent, compressible, reactive Euler equations,

$$
\begin{aligned}
& \frac{\partial \rho}{\partial t}+\nabla \cdot(\rho \mathrm{U})=0, \\
& \frac{\partial(\rho \mathrm{U})}{\partial t}+\nabla \cdot(\rho \mathrm{UU})+\nabla P=0, \\
& \frac{\partial E}{\partial t}+\nabla \cdot((E+P) \mathrm{U})+q \rho \dot{w}=0, \\
& \frac{\partial(\rho Y)}{\partial t}+\nabla \cdot(\rho Y \mathrm{U})-\rho \dot{w}=0,
\end{aligned}
$$

where $\rho$ is the mass density, $U$ is the velocity, $E$ is the energy density, $P$ is the pressure, $Y$ is the mass fraction of a reactant, $\dot{w}$ is the reaction source term, and $q$ is the energy release. The equation of state is that of an ideal gas

$$
P=\frac{\rho R T}{M}, \quad E=\frac{P}{(\gamma-1)}+\frac{\rho U^{2}}{2},
$$


where $\gamma$ is the adiabatic index, $R$ is the universal gas constant, and $M$ is the molecular weight. The chemical source term describes the first-order Arrhenius kinetics

$$
\frac{d Y}{d t} \equiv \dot{w}=-A \rho Y \exp \left(-\frac{Q}{R T}\right)
$$

where $A$ is the pre-exponential factor, and $Q$ is the activation energy. The reaction rate $\dot{w}$ is proportional to $\rho$ to account for the mostly binary nature of chemical reactions taking place in real combustion systems.

We model mixtures initially at temperature $T_{0}=$ 293K and pressure $P_{0}=0.2 \mathrm{~atm}$, and adopt the value of activation energy $Q / R T_{0}=38.2$ according to experimental data for ethylene-oxygen. ${ }^{13}$ The value of $A=7 \times 10^{10}$ was selected to match experimental size of a detonation cell in a stoichiometric ethyleneoxygen diluted with argon. ${ }^{14}$ Detailed thermodynamic calculations of the ZND and detonation shock parameters using ${ }^{12}$ show that $\gamma$ is systematically higher for ethylene-oxygen systems diluted with argon than for systems with nitrogen dilution. ${ }^{1}$ The calculations also show that $\gamma$ is not constant inside the reaction zone but decrease slightly toward the Chapmen-Jouguet point where reactions come to completion. For simulations, we select $M$ and $\gamma$ as average values obtained from detailed thermodynamic calculations, and then adjust the energy release $q$ to match the experimentally measured CJ velocity $D$ of the system. The choice of $q$, $M$, and $\gamma$ fixes all other thermodynamical parameters of the model system including the post-shock temperature $T_{s}$, and the reduced activation energy $Q / R T_{s}$. In our calculations the chemical model (3) is the same for all mixtures, whereas $\gamma, M$, and $q$ are varied to reflect changes in system's dilution.

The Euler equations (1) are integrated using an explicit, second-order, Godunov type, adaptive-meshrefinement code ALLA described in. ${ }^{15}$ Euler fluxes are evaluated by solving a Riemann problem at cell interfaces. Mesh refinement is based on a Fully Threaded Tree (FTT) discretization of the computational domain. The mesh is dynamically refined around shocks, flame fronts, and in regions of large gradients of density, pressure, or composition. An example of an FTT adaptive mesh is shown on a cover page together with the pressure field in one of the calculations. The chemical reaction part (3) is a set of ordinary differential equations for $Y$, one equation per computational cell. These equations are integrated assuming constant mass density during a time step. The code has been previously used for various combustion and detonation problems including shock-flame interaction, deflagration-to-detonation transition, and detonation wave structure, and tested extensively.

The computational domain modeled a part of a rectangular detonation tube used in experiments described in the next section below. For mixtures diluted with nitrogen and displaying irregular behavior, the width of the computational domain was equal to the width of the tube $=15 \mathrm{~cm}$. For mixtures diluted with argon, the detonation cell size $<1 \mathrm{~cm}$ was much less than the tube width, and the computational domain width was decreased to accommodate only $\sim 3$ detonation cells within the computational domain. In all calculations, the length of the domain was two times larger then its width. Upper and lower boundaries of the domain were modeled as solid walls, and zero gradient inflow/outflow was imposed on the left and right boundaries. A planar ZND detonational wave propagating in X-direction, or left-to-right, was initially placed approximately one detonation cell length away from the right boundary. The inflow velocity equal to the detonation speed $D$ was imposed on the right boundary in order to keep the detonation front at the same position within the computational domain. A $10 \%$ temperature perturbation was introduced in a small area inside the detonation structure at approximately $\frac{1}{4}$ of the width from the lower boundary. The perturbation quickly spread behind the way in both $\mathrm{X}$ and $\mathrm{Y}$-directions causing a rapid development of a cellular structure.

Numerical resolution of adaptive simulations can be characterized by specifying minimum and maximum levels of refinement of the computational mesh, $l_{\min }$ and $l_{\max }$. Cell size $\Delta x$ is related to the level of refinement $l$ as $\Delta x=L / 2^{l}$, where $L$ is the length of the computational domain and $l_{\min } \leq l \leq l_{\max }$. The simulations were initiated with $l_{\min }=l_{\max }=6$. The resolution was then gradually increased until the detonation cell structure recorded by the numerical maximum pressure soot foils stopped changing with the resolution. Additional resolution tests were conducted by re-running simulations with different resolutions and comparing results for the same moments of time. The issue of numerical resolution is revisited and further discussed throughout this paper.

\section{Numerical simulations}

\section{Regular and semi-regular cellular detonations}

Calculations for ethylene-oxygen diluted with argon (Case 1) were carried out for the system $\mathrm{C}_{2} \mathrm{H}_{4}+$ $3 \mathrm{O}_{2}+11.2 \mathrm{Ar}$ for which $M=35, \gamma=1.313$ and $q M / R T_{0}=53.0$ were selected following the procedure described in the previous section. With these parameters, the CJ detonation velocity is $D=1.7 \mathrm{~km} / \mathrm{s}$, the post-shock temperature $T_{s}=1711 \mathrm{~K}$, and the reduced activation energy is $Q / R T_{s}=6.54$. The length of the computational domain was taken $L=3.5 \mathrm{~cm}$. Numerical resolution was varied from $l_{\min }=l_{\max }=6$ to $l_{\min }=6, l_{\max }=13$. Results of calculations for Case 1 are presented in Fig.1 to 3.

Fig. 1 shows a cycle of the detonation wave propagation computed using highest resolution $l_{\max }=13$. The cycle duration is $t_{c} \simeq 6 \mu$ s and corresponds to a 

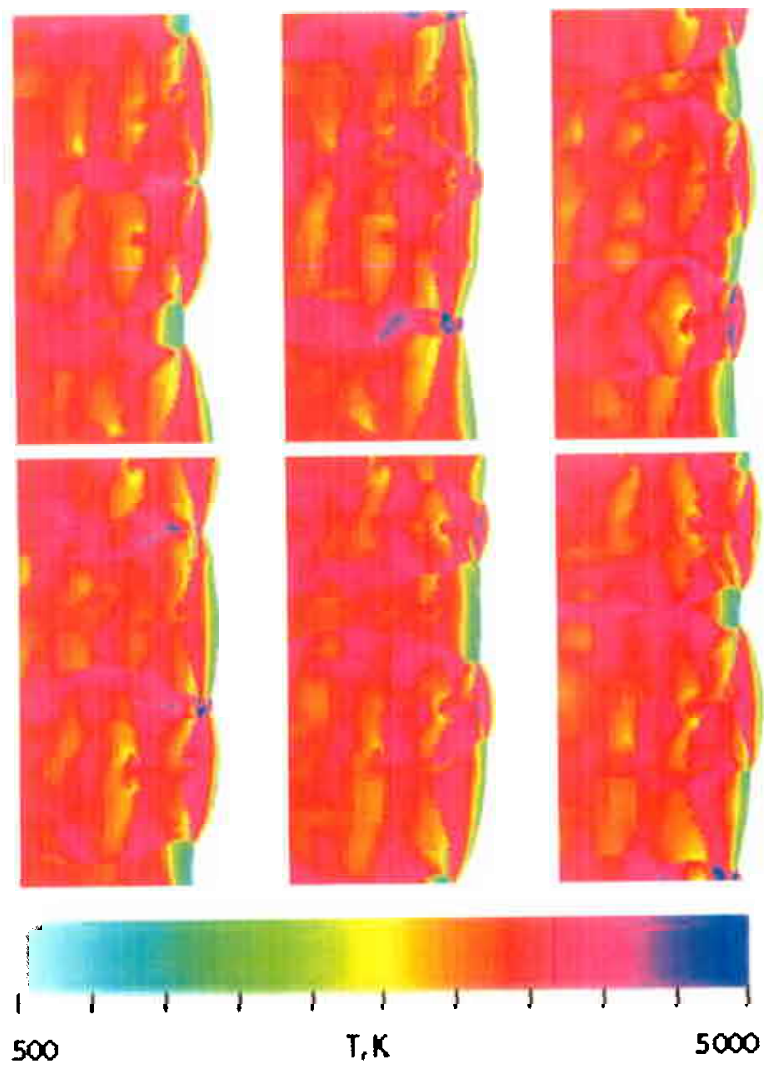

Fig. 1 Temperature field inside the reaction zone of a detonation front in $\mathrm{C}_{2} \mathrm{H}_{4}+3 \mathrm{O}_{2}+11.2 \mathrm{Ar}$. Snapshots correspond to times $t=17.76,17.86$, and 17.98 $\mu$ s (first raw) and $t=18.10,18.22$, and $18.31 \mu \mathrm{s}$ (second raw) since the beginning of a simulation. The figure shows one detonation cycle which lasts $\simeq 0.6$ $\boldsymbol{\mu s}$.
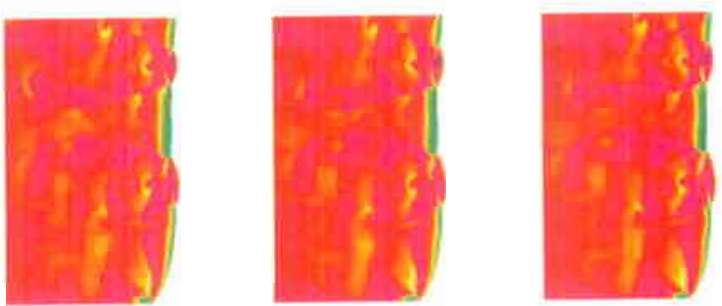

Fig. 2 Structure of a detonation front (temperature fleld) computed for $t=18.22 \mu \mathrm{s}$ using three numerical resolutions $l_{\max }=11,12,13$ (from left to right).
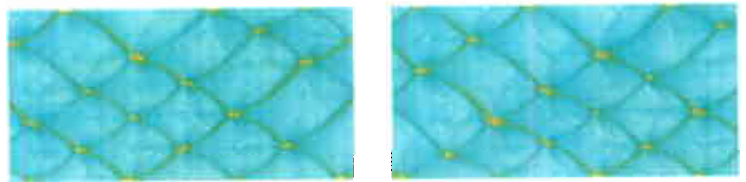

Fig. 3 Numerical soot foils recording maximum pressure for the Case 1. Recorded at resolutions $l_{\max }=11$ and 12. No signifcant differences can be observed between the resolutions. cell length $D \cdot t_{c} \simeq 1 \mathrm{~cm}$.

Fig. 2 shows the reaction zone structure for the same moment of time $t=18.22$ calculated with three different maximum resolutions, $l_{\max }=11,12$, and 13 . The position of the front and the large scale details of the flow are practically indistingushable at all resolutions. The $l_{\max }=12$ is more than sufficient for reproducing the cellular structure for this mixture. In fact, the $l_{\max }=11$ resolution is enough. The small scales structures can be seen to emerge when the resolution is increased. However, due to a relatively weak coupling between the chemical reaction and fluid flow they do not grow fast enough to affect the overall characteristics of the detonation.

Fig. 3 shows the numerical soot foils for the detonation Case 1 recorded at two different numerical resolutions. The foils show a quasi-periodic structure with a single level of detonation cells. A very slight variability of cell sizes can be seen so that Case 1 should perhaps be characterized as marginally irregular. Decreasing the reduced activation energy from $Q / R T_{\mathrm{s}}=6.54$ to $Q / R T_{s}=6.0$ virtually eliminates the cell variability (Fig. 4).
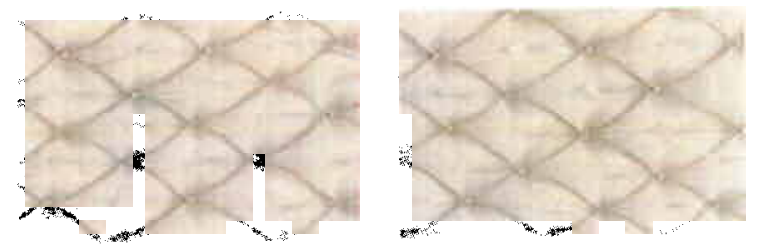

Fig. 4 Numerical soot foils for the case of a regular detonation with $Q / R T_{s}=6.0$. Computed with resolutions $l_{\max }=10$ and 11 .

\section{Irregular cellular detonation}

Calculations for ethylene-oxygen diluted with nitrogen (Case 2) were carried out for a system $\mathrm{C}_{2} \mathrm{H}_{4}+$ $3 \mathrm{O}_{2}+11.2 \mathrm{~N} 2$ for which the parameters $M=28.0$, $\gamma=1.228$ and $q M / R T_{0}=34.0$ were selected following the procedure described in previous section. The CJ detonation velocity in this system is $D=1.79$ $\mathrm{km} / \mathrm{s}$, the post-shock temperature $T_{\mathrm{s}}=1285 \mathrm{~K}$, and the reduced activation energy is $Q / R T_{\mathrm{s}}=8.71$. The length of the computational domain was taken $L=30$ $\mathrm{cm}$ so that the width of the computation domain was equal to the width of the detonation tube used in the experiments. ${ }^{1}$ Numerical resolution was varied from $l_{\min }=l_{\max }=6$ to $l_{\min }=6, l_{\max }=13 . \mathrm{Re}$ sults of calculations for Case 2 are presented in Fig. 5 and 6. Change of a dilutent from argon to nitrogen decreases the adiabatic index of matter from $\gamma=1.313$ to $\gamma=1.228$ and this reduces the post-shock temperature $T_{s}$ by almost $40 \%$. The resulting higher value of the effective reduced activation energy $Q / R T$, causes a pronounced irregular behavior of a detonation front. The reaction zone shows filaments of unburned material along the front, some evidence of pockets of 
unreacted material, and the emergence of a secondary detonation cell structure. Decrease of the post-shock temperature also leads to the increase of an induction time behind the shock wave, and the increase of the detonation cell size from $\simeq 0.7 \mathrm{~cm}$ for Case 1 to $\simeq 5 \mathrm{~cm}$ for Case 2. Exact determination of a detonation cell size in Case 2 is difficult due to large variations among individual cells. Cell size obtained in the simulation is consistent with the experimental value $\simeq 5 \mathrm{~cm}$ found in. 1

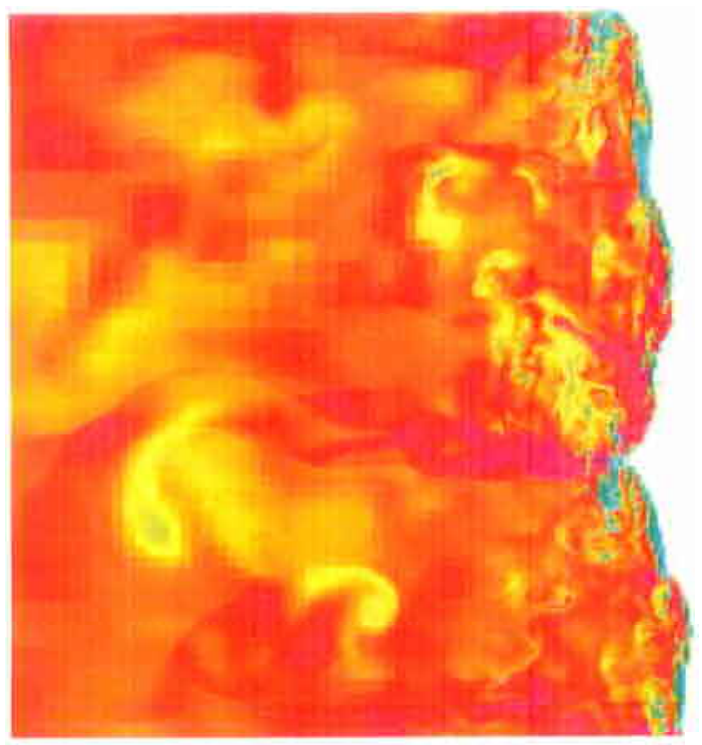

Fig. 5 Temperature field inside the reaction zone of a detonation front in $\mathrm{C}_{2} \mathrm{H}_{4}+3 \mathrm{O}_{2}+11.2 \mathrm{~N} 2$ at time $t=11.63 \mu \mathrm{s}$. Resolution $l_{\max }=13$.

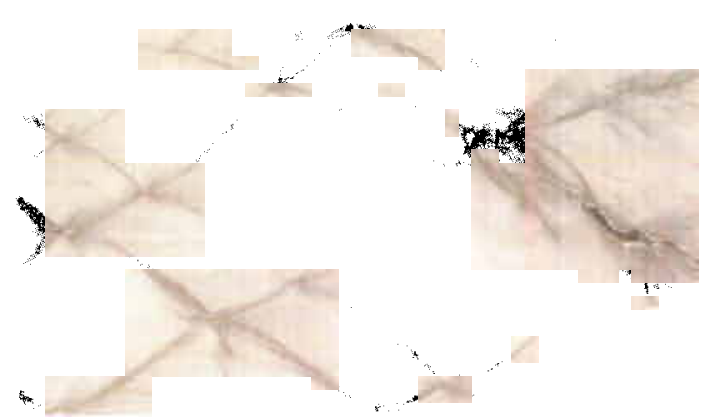

Fig. 6 Numerical soot foils for the case of an irregular detonation in $\mathrm{C}_{2} \mathrm{H}_{4}+3 \mathrm{O}_{2}+11.2 \mathrm{~N} 2$.

\section{Highly irregular cellular structure}

Further increase of the effective activation energy leads to even more erratic behavior of the front with pronounced pockets of unreacted matter and secondary cell structures. Fig. 7 shows the temperature field inside the reaction zone of a detonation with $Q / R T_{s}=10.5$. This detonation was obtained by decreasing the Case $2 \gamma$ to lower value $\gamma=1.16$ and changing $q$ accordingly to keep the detonation velocity unchanged. Numerical soot foil record for this deto- nation, Fig. 8, shows a more pronounced secondary cellular structures than these obtained in Case 2.

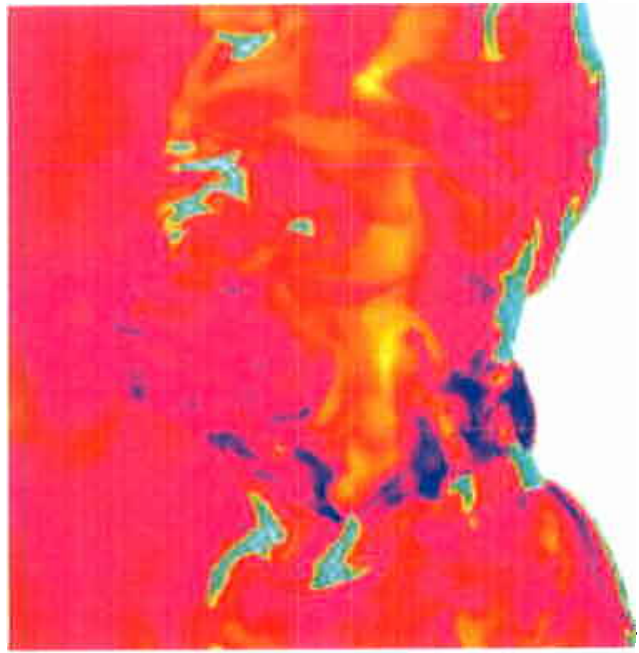

Fig. 7 Temperature field inside the reaction zone structure for a highly irregular detonation with $Q / R T_{s}=10.5$. Resolution $l_{\max }=11$.

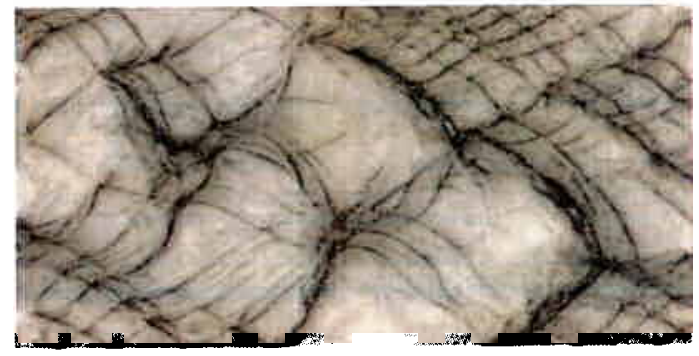

Fig. 8 Numerical soot foils for the case of an irregular detonation with $Q / R T_{s}=10.5$.

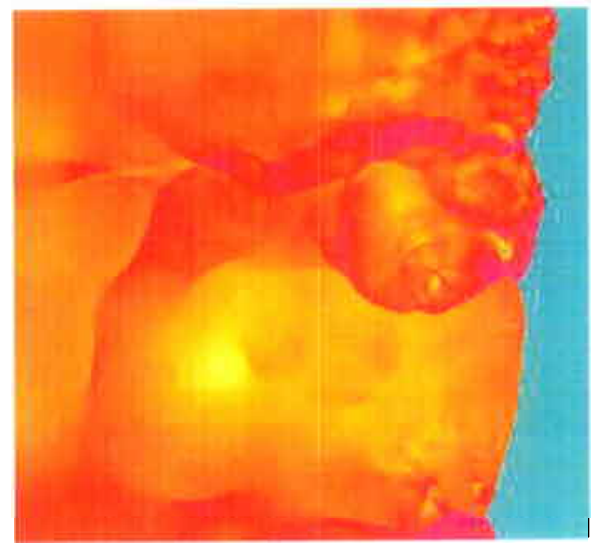

$\mathbf{P}$

Fig. 9 Pressure field inside the reaction zone structure for a highly irregular detonation with $Q / R T_{a}=10.5$. Resolution $l_{\max }=11$. A localized explosion is seen in the vicinity of collisions of two transverse waves.

Fig. 9 presents a pressure field for one moment of 
time during the propagation of a $Q / R T_{s}=10.5$ detonation and shows a blast wave from a strong localized explosion which originated from the region near the collision of two transverse waves. These explosions are not observed in less unstables cases discussed above. Calculations show that localized explosions begin to appear in behind the leading shock wave of highly unstable detonations with approximately $Q / R T,>8-9$.

\section{Discussion}

This numerical study was motivated by recent experiments carried at the Graduate Aeronautical Laboratory at Caltech in which a simultaneous visualization of chemical species and of density gradients in the reaction zones of propagating detonations has been achieved??. Experiments were carried out in a $4.2 \mathrm{~m}$ long channel with cross-section $152 \times 18 \mathrm{~mm}$ at initial pressure 150 Torr.? Ruby laser schlieren and planar laser induced fluorescence of the $\mathrm{OH}$ radical ${ }^{?}, 17,18$ were used to obtain separate images of the shock configurations and reaction front structure for a single experiment. The two images were then superimposed by post-processing with a time between images of less then 800 ns. Soot foil data were also collected for some of the same systems.

According to experimental data, ${ }^{14}$ the detonation in $\mathrm{C}_{2} \mathrm{H}_{4}+3 \mathrm{O} 2+11.2 \mathrm{Ar}$ should be regular. Simulations presented here are consistent with the experiment. The numerical detonation cell size derived from Fig. 3 $\simeq 0.6 \mathrm{~cm}$ is in agreement with with experimental value reported in. ${ }^{14}$ Experimental images of the detonation structure for a detonation in $\mathrm{C}_{2} \mathrm{H}_{4}+3 \mathrm{O}_{2}+11.2 \mathrm{Ar}$ are not available. However, a detonation in the narrow channel for a weakly unstable detonation in an argon-diluted hydrogen-oxygen mixture with a similar value of $Q / R T_{s} \simeq 6$ was studied in. ${ }^{17,18}$ Experimental images are shown in Fig. 10. The cell width for this mixture is on the order of $25-35 \mathrm{~mm}$ and the separation between the leading shock and $\mathrm{OH}$ front can be up to $5 \mathrm{~mm}$ depending on the location within the cell. We see features in the schlieren inuage that are very similar to those observed by previous researchers. ${ }^{19-23}$ Triple point configurations in general appear to be of the weak type, irrespective of the location in the cell cycle. The keystone features seen in our previous study ${ }^{18}$ are also apparent in Fig. 10b. From the overlay image in Fig. 10 (c), it can now be quite clearly seen that the shear layer originating at the intersection of the main front and transverse wave separates reacted and unreacted gas, forming the boundary of the keystone.

It should be noted that due to rapid variations of the detonation structure, an agreement between observed and calculated transient features depends on the moment of time chosen for a comparison. A 2D numerical simulation with reduced activation energy $Q / R T_{s}=6$ shown in the lower right frame of Fig. 10. The temper-
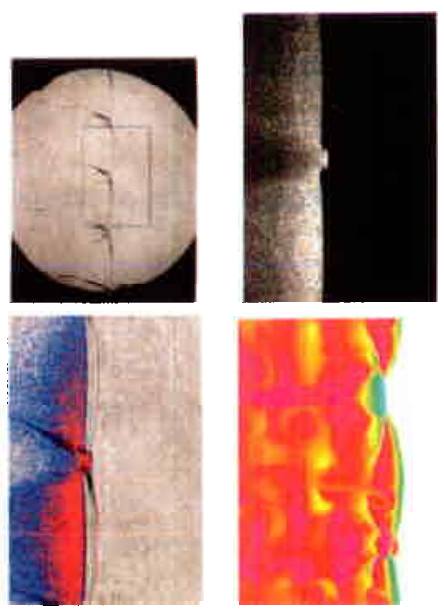

Fig. 10 Images of a detonation front propagating from left to right in $2 \mathrm{H}_{4}-\mathrm{O}_{2}-17 \mathrm{Ar}, P_{1}=20 \mathrm{kPa}$ in the narrow channel. Schlieren image - upper left frame. The box shows the location of the corresponding $\mathrm{OH}$ fluorescence image shown in upper right frame. Superimposed schlieren and fluorescence image. PLIF image is $60 \mathrm{~mm}$ high - lower left. The effective non-dimensional activation energy is estimated to be $Q / R T_{a}=6$. Lower right image shows temperature distribution for a simulation with reduced activation energy $Q / R T_{s}=6$ at time $t=86.5 \mu \mathrm{s}$.

ature distribution corresponds qualitatively to the $\mathrm{OH}$ distribution in the experimental PLIF images shown in the same figure. In the bottom right, a keystone shaped feature similar to that observed in experiments is evident in the numerical solution. Experimental soot foils Fig. 11 were obtained for the detonation shown in Fig. 10 by mounting aluminum sheets covered in a thin layer of soot to the side walls of the test section and show tracks which correspond to the triple point tracks in the regular front.? They agree well with the calculated soot foils shown in Fig. 4.

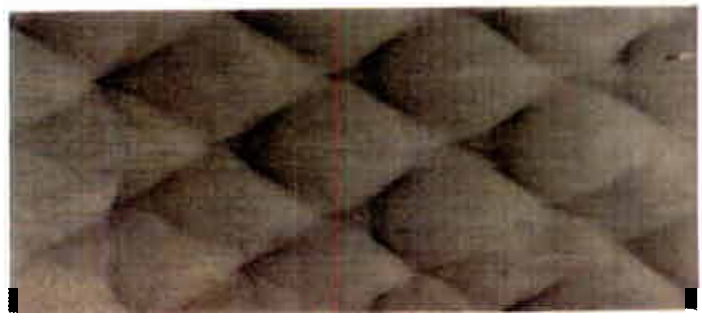

Fig. 11 Experimental soot foil in $2 \mathrm{H}_{2}-\mathrm{O}_{2}-17 \mathrm{Ar}$, $P_{o}=20 \mathrm{kPa}$.

Unstable detonations in mixtures with reduced activation energy higher than about $Q / R T_{s} \simeq 8$ have a significantly more complex structure. This is manifested by a wrinkled shock front and density disturbances on multiple scales as seen in the experimental schlieren images. The reaction front evident in the PLIF images is more distorted and disorganized than in the regular case. A typical example is Fig. 12 which 
shows the detonation structure in $\mathrm{C}_{2} \mathrm{H}_{4}-3 \mathrm{O}_{2}-10.5 \mathrm{~N}_{2}$ mixture. This figure should be compared to Fig. 7 and Fig. 5 showing the same behavior of numerically calculated irregular detonations. Soot foils for the irregular mixtures (see Fig. 13) show structure over a range of scales in contrast to the single dominant scale observed in regular mixtures. In some mixtures, substructure is observed. Numerical soot foils of irregular detonations show qualitatively the same features (compare to Fig. 6 and 8 to Fig. 13).
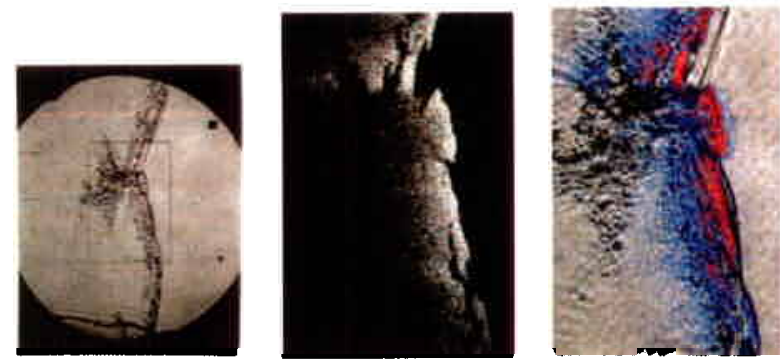

Fig. 12 Images of a detonation front propagating from left to right in $\mathrm{C}_{2} \mathrm{H}_{4}-3 \mathrm{O}_{2}-10.5 \mathrm{~N}_{2}, P_{1}=20 \mathrm{kPa}$ in the narrow channel. (a) Schlieren image. The box shows the location of the corresponding $O H$ fluorescence image shown in (b). (c) Superimposed schlieren and fluorescence image. PLIF image is $30 \mathrm{~mm}$ high.

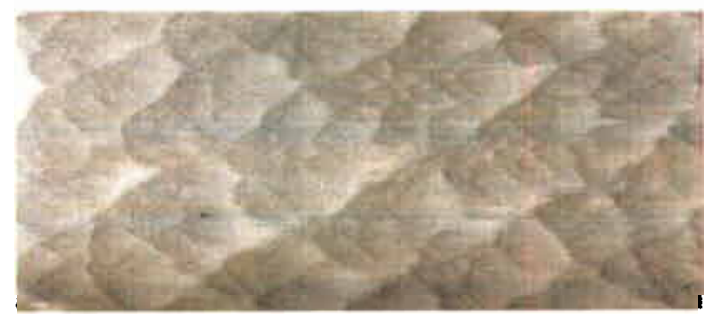

Fig. 13 Experimental soot foil in $\mathrm{C}_{2} \mathrm{H}_{4}-3 \mathrm{O}_{2}$ $10.5 \mathrm{~N}_{2}, P_{1}=20 \mathrm{kPa}$.

In highly irregular fronts, localized explosions are observed in both numerical simulations with lowest values of $\gamma$. and in experiments in mixtures with the highest degree of instability. Fig. ?? shows a strong localized explosion which originated from the region near the collision of two transverse waves, Fig. 9 in a mixture with artificially decreased $\gamma$ which is low compared to $\gamma$ predicted for ethylene-oxygen diluted with nitrogen (see previous section). Localized explosions were not experimentally observed in ethylene-oxygennitrogen mixtures, as well. Numerical simulations are thus consistent with experimental data. In experiments, localized explosions were observed in $\mathrm{C}_{3} \mathrm{H}_{8}$ $5 \mathrm{O}_{2}-9 \mathrm{~N}_{2}$ (Fig. 14 with the highest degree of instability. Experimental images (Fig. 14) show regions of high fluorescence intensity in the region of transverse wave interaction at the apex of the cell. This explosive interaction contrasts with the mild collision process that occurs in regular mixtures.
In highly irregular fronts, localized explosions are observed in both numerical simulations and experiments. The pressure field for a highly irregular mixture shows a strong localized explosion which originated from the region near the collision of two transverse waves, Fig. ??. Experimental images (Fig. 14) show regions of high fluorescence intensity in the region of transverse wave interaction at the apex of the cell. This explosive interaction contrasts with the mild collision process that occurs in regular mixtures and mildly irregular mixtures.

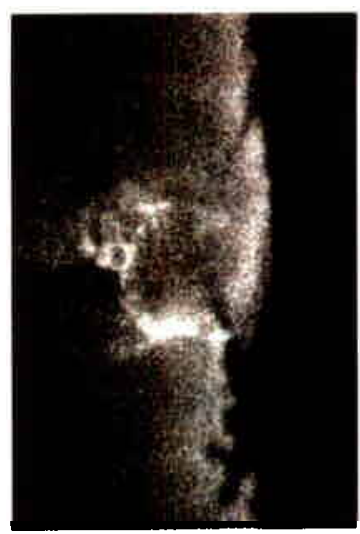

Fig. 14 Experimental OH fluorescence image of detonation front propagating in $\mathrm{C}_{3} \mathrm{H}_{8}-\mathbf{5 O}_{2}-9 \mathrm{~N}_{2}$, $P_{1}=20 \mathrm{kPa}$. Image height is $30 \mathrm{~mm}$.

\section{Conclusions}

In this papewr we carried out numerical simulations of detonations in ethylene-oxygen mixtures with varying dilution. The chemical model was the same for all mixtures considered in this study. Effects of dilution were modeled by varying the adiabatic index of matter $\gamma$. Variations of $\gamma$ due to variations in the nature of dilutent were estimated based on detailed thermodynamical calculations of ZND detonation in considered mixtures. Due to a strong sensitivity of the post-shock temperature to the equation of state and $\gamma$, small variations of $\gamma$ lead to large variations of the post-shock temperature $T_{s}$ and the corresponding reduced activation energy $Q / R T_{s}$. The latter quantity controls the degree of instability of chemical - fluid dynamics coupling inside the detonation structure and the behavior of the system.

By varying $\gamma$ in the narrow range $1.35>\gamma>1.15$, we were able to qualitatively reproduce a wide range of behaviors of a detonation front. Higher values of $\gamma \simeq 1.3$ typical of argon dilutions lead to $Q / R T_{s} \simeq 6$ and a regular behavior of cellular detonations. In our simplified model, dilution with nitrogen decreases $\gamma$ and increases the reduced activation energy to values around $Q / R T_{s} \simeq 8-9$, leading to irregular behavior of a detonation. There are a number of features obtained in numerical simulations of irregular detonations which 
are qualitatively similar to those observed in experimental PLIF images, including pronounced filaments on the front, pockets of unreacted material behind the front, and distorted keystone features. The cellular substructure is frequently found in the experiments near the upstream apex of the main cell, although smaller cells can sometime be seen throughout some of the larger cells. Numerical results show qualitatively similar behavior. Finally, numerical simulations for highly irregular cases with low $\gamma$ show strong localized explosions which originate from the regions near collision of two transverse waves. Similar behavior has been experimentally observed for highly irregular detonations.

Results of this study indicate that the effect of the equation of state and the influence of dilution on the adiabatic index of matter and the post-shock temperature $T_{s}$ may be an impotant mechanisms controlling the behavior of cellular detonations, and a wide range of detonation behaviors can be linked to rather modest variations of $\gamma$. Higher $\gamma$ leads to higher $T_{s}$ and a weaker coupling between chemical reactions and fluid dynamics. Smaller $\gamma$ (softer equation of state) lead to smaller $T_{s}$, increased instability, and a less regular behavior of a detonation. Within the framework of a simplified Arrhenius kinetic model with a single progress variable, the variations of $\gamma$ due to variations in dilution seem to be sufficient for qualitatively reproducing a wide range of detonation behaviors observed in detonation tube experiments.

\section{References}

${ }^{1}$ Austin, J.M., "The role of instability in gaseous detonation," PhD thesis, California Institute of Technology, Pasadena, California, June 2003.

${ }^{2}$ S. Taki, S. and Fujiwara, T.", 1978, "Numerical Analysis of Two-Dimensional Nonsteady Detonations," AIAA Journal, 16, 73-77.

${ }^{3}$ Oran, E.S., Young, T.R., Boris, J.P., Picone, J.M., Edwards, D.H., 1982, "A Study of Detonation Structure: The Formation of Unreacted Gas Pockets," in 19th Symp. (Int.) on Combustion, 573-582, The Combustion Institute.

${ }^{4}$ Williams, D.N., Bauwens, L., Oran, E.S., 1996, "Detailed structure and propagation of thre-dimensional detonations," Proc. Combust. Inst. 26, 2991-2998.

${ }^{5}$ Bourlioux, A. and Majda, A.J., 1992, "Theoretical and numerical structure for unstable two-dimensional detonations," Combust. Flame, 90, 211-229.

${ }^{6}$ Gamezo, V.N., Desbordes, D., Oran, E.S., 1999, "Twodimensional reactive flow dynamics in cellular detonation waves," Shock Waves, 9, 11-17.

${ }^{7}$ Eckett, C.A., "Numerical and Analytical Studies of the Dynamics of Gaseous Detonations," PhD thesis, California Institute of Technology, Pasadena, California, September 2000.

${ }^{8}$ Sharpe, G.I. and Falle, S.A.E., 2000, "Two-dimensional numerical simulations of idealized detonations," Proc. R. Soc. London, A 456, 2081-2100.

${ }^{9}$ Gamezo, V.N, Khokhlov, A.M., Oran, E.S., 1999, "Secondary detonation cells in systems with high activation energy," in Proceedings of the Seventeenth International Colloquium an the Dynamics of Explosions and Reactive Systems, Universitat Heidelber, Heidelberg, Germany, IWR.
${ }^{10}$ Gamezo, V.N., Vasiliev, A.A., Khokhlov, A.M., Oran, E.S., 2000 , "Fine cellular structure produced by marginal detonations," Proceedings of the Combustion Institute, 28, 611-617.

${ }^{11}$ Short, M. and and Stewart, D.S., 1998, "Cellular detonation stability. Part 1. A normal-mode linear analysis," $J$. Fluid Mech., 368, 229-262.

${ }^{12}$ Reynolds, W.C., 1986 "The Element Potential Method for Chemical Equilibrium Analysis: Implementation in the Interactive program STANJAN", "Stanford University, Dept of Mech. Engng".

${ }^{13}$ Drummond, L.J., 1968, "Shock-initiated exothermic reactions: The oxydation of ethylene," Aust. J. Chem., $212641-$ 2648.

${ }^{14}$ Strehlow, R.A., Engel, C.D., 1968, "Transverse Waves in Detonations: II. Structure and Spacing in $\mathrm{H}_{2}-\mathrm{O}_{2}, \mathrm{C}_{2} \mathrm{H}_{2}-\mathrm{O}_{2}$, $\mathrm{C}_{2} \mathrm{H}_{4}-\mathrm{O}_{2}$, and $\mathrm{CH}_{4}-\mathrm{O}_{2}$ Systems," AIAA Journal, 7, 492-323.

${ }^{15}$ Khokhlov, A.M., 1998, "Fully threaded tree algorithms for adaptive mesh fluid dynamics simulations," J. Comp. Physics, 143, 519-543.

${ }^{16}$ Shepherd, J.E., Pintgen, F., Austin, J.M., Eckett, C.A., 2002, "The structure of the detonation front in gases," AIAA paper 2002-0773.

${ }^{17}$ Pintgen, F., 2000, "Laser-Optical Visualization of Detonation Structures," "Diplom Arbeit", school = "Lehrstuhl für Thermodynamik: Technische Universität München / Graduate Aeronautical Laboratories: California Institute of Technology".

${ }^{18}$ Pintgen, F., Eckett C.A., Austin, J.M., and Shepherd, J.E., 2003, "Direct Observations of Reaction Zone Structure in Propagating Detonations," Combust. Flame, 133, 211-229.

${ }^{19}$ Voitsekhovskii, B.V., Mitrofanov, V.V., Topchian, M.E., 1963, "Struktura fronta detonastii i gaza," Akad. Nauk., SSSR, Novosibirsk.

${ }^{20}$ Strehlow, R.A., 1964, "Reactive gas Mach stems," Phys. Fluids, 7, 908-909

${ }^{21}$ Oppenheim, A.K., Smolen, J.J., Zajac, L.J., 1968, “ Vector polar method for the analysis of wave intersections," Combust. Flame, 12, 63-76.

${ }^{22}$ Urtiew, P.A., 1970, "Reflections of wave intersections in marginal detonations," Astronautica Acta, 15, 335-343.

${ }^{23}$ D. H. Edwards, D.H., Hooper, G., Meddins, R.J., 1972, "Instabilities in the reaction zones of detonation waves," Astronautica Acta, 17, 475-485. 